\title{
THE BILINEAR RELATION ON OPEN RIEMANN SURFACES $\left({ }^{1}\right)$
}

\author{
BY \\ ROBERT D. M. ACCOLA(2)
}

I. Introduction. With the foundations of the theory of square integrable differentials on Riemann surfaces firmly laid (Ahlfors, $[2 ; 3]$ ), it is natural to ask how much of the theory of closed surfaces can be generalized to open surfaces in terms of square integrable differentials. The Hilbert space of square integrable harmonic differentials seems an appropriate context in which to carry over the algebraic results which hold on closed surfaces. This paper investigates such questions with particular attention to generalizing Riemann's bilinear relation.

We begin with a Riemann surface $W$ and the class $\Gamma_{h}$ of all square integrable harmonic differentials defined on $W$. A harmonic differential is locally the differential of a function whose real and imaginary parts are harmonic functions. Suppose a differential $\omega$ has the local representation $\omega=a d x+b d y$. If we define the conjugate $\omega^{*}$ of $\omega$ by $\omega^{*}=-b d x+a d y$, then we can equivalently define $\omega$ to be harmonic by requiring that $\omega$ and $\omega^{*}$ be closed differentials. Note that $\omega^{* *}=-\omega$. To say that $\omega$ is square integrable means that the integrals

$$
\iint_{K}\left(|a|^{2}+|b|^{2}\right) d x d y
$$

are bounded as $K$ ranges over all compact subsets of $W$. The Dirichlet norm of $\omega$, denoted $\|\omega\|$, is defined so that $\|\omega\|^{2}$ is the supremum of such integrals. If $\sigma=a^{\prime} d x+b^{\prime} d y$ we define the inner product $(\omega, \sigma)$ by

$$
(\omega, \sigma)=\iint_{W}\left(a \bar{a}^{\prime}+b \bar{b}^{\prime}\right) d x d y .
$$

The fundamental result which makes this type of analysis fruitful is that $\Gamma_{h}$ is a Hilbert space.

Now suppose that $c$ is an oriented rectifiable path on $W$. Then we consider the integral of $\omega$ along $c$. If $\omega$ is closed and $c$ is a cycle, then $\int_{c} \omega$ depends only

Presented to the Society, April 24, 1959; received by the editors September 14, 1959.

(1) Most of the work on the paper was sponsored by the Air Force Office of Scientific Research under contract number AF 18(600)-1461.

(2) The author is indebted to Professor L. V. Ahlfors for allowing him access to the manuscript of the book on Riemann surfaces by Professors Ahlfors and Sario. 
on the homology class of $c$, and $\int_{c} \omega$ is called the period of $\omega$ along $c$. We say that $c$ is a dividing cycle if for any compact set $K$, there is a cycle $c^{\prime}$ entirely outside $K$ such that $c$ is homologous to $c^{\prime}$. If $c$ is composed of Jordan curves, then this definition is easily seen to imply that $c$ divides $W$ into at least two pieces.

By a finite Riemann surface, we shall mean the interior of a compact bordered surface. If $W$ is the finite surface, $\bar{W}$ will denote the bordered surface of which $W$ is the interior. The dividing cycles of $W$ are cycles homologous to sums of oriented boundary contours. We will consider boundary contours of $\bar{W}$ oriented so as to agree with the orientation induced by the conformal structure of $W$.

If $\Gamma_{p}$ is a class of differentials, then $\Gamma_{p}^{*}$ is the class of differentials whose conjugates are in $\Gamma_{p}$. If $A$ is a Riemann surface, we use the notation $\Gamma_{p}(A)$ to denote differentials of class $\Gamma_{p}$ defined on $A$. If the surface in question is obvious we will use the shorter notation $\Gamma_{p}$.

We now distinguish several classes of harmonic differentials on an open surface $W$.

$\Gamma_{h e}$ : exact harmonic differentials; that is, differentials of functions whose real and imaginary parts are harmonic on all of $W$.

$\Gamma_{h o}$ : the orthogonal complement in $\Gamma_{h}$ of $\Gamma_{h e}^{*}$. If $W$ is a finite surface, then it can be shown that $\omega \in \Gamma_{h o}$ can be extended to be harmonic on $\bar{W}$ and the extension will be zero along the contours of $\bar{W}\left({ }^{3}\right)$.

$\Gamma_{h s e}$ : semi-exact differentials; that is, differentials whose periods along the dividing cycles are all zero.

$\Gamma_{h m}$ : harmonic measures; that is, the orthogonal complement in $\Gamma_{h}$ of $\Gamma_{l s s e}^{*}$. If $W$ is a finite surface then $\Gamma_{h m}=\Gamma_{h o} \cap \Gamma_{h e}$. In this case $\Gamma_{h m}$ is the collection of differentials of linear combinations of harmonic measures of $\bar{W}$.

$\Gamma_{a}$ : analytic differentials. Note that for an analytic differential $\alpha$ it is true that $\alpha^{*}=-i \alpha$.

$\Gamma_{a e}$ : exact analytic differentials.

$\Gamma_{a S}$ : analytic Schottky differentials; that is, the class $\Gamma_{a} \cap C L\left(\Gamma_{h o}+\Gamma_{h o}^{*}\right)$. It can be shown that $\Gamma_{a S}$ is the orthogonal complement of $\Gamma_{a e}$ in the space of analytic differentials.

By definition we thus have the orthogonal decompositions:

$$
\begin{aligned}
& \Gamma_{h}=\Gamma_{h o}+\Gamma_{h e}^{*}=\Gamma_{h o}^{*}+\Gamma_{h e}, \\
& \Gamma_{h}=\Gamma_{h m}+\Gamma_{h s e}^{*}=\Gamma_{h m}^{*}+\Gamma_{h s e .}
\end{aligned}
$$

Also we have $\Gamma_{h e} \subset \Gamma_{h s e}$ and $\Gamma_{h o} \subset \Gamma_{h s e}$.

If $W \in O_{H D}$, that is, if $W$ admits no nonconstant harmonic functions whose all $t$.

$\left.{ }^{3}\right) \omega=a d x+b d y$ is said to vanish along a differentiable arc $z=z(t)$ if $a x^{\prime}(t)+b y^{\prime}(t)=0$ for 
differential has finite Dirichlet norm, then $\Gamma_{h}=\Gamma_{h o}=\Gamma_{h s e}$. Since it is well known that parabolic surfaces are of class $O_{H D}$, we can say the same of them.

We consider further the periods of harmonic differentials. If $c$ is a cycle, then $\int_{c} \omega$ can be viewed as a linear functional on $\Gamma_{h}$. It can be shown to be a bounded linear functional. By standard Hilbert space theory, there exists a harmonic differential $\sigma(c)$ so that $\int_{c} \omega=\left(\omega, \sigma^{*}(c)\right)$. Such a $\sigma(c)$ is unique, real, of class $\Gamma_{h o}$, and depends only on the homology class of $c$. Applying the same result to $\Gamma_{a}$ yields a unique analytic Schottky differential $\psi(c)$ which reproduces the periods of analytic differentials; that is, if $\alpha \in \Gamma_{a}$ then $\int_{c} \alpha$ $=(\alpha, \psi(c))$. It follows easily that $\sigma(c)=-2 \operatorname{Im}\{\psi(c)\}$ and $\sigma^{*}(c)=2 \operatorname{Re}\{\psi(c)\}$, for $\sigma^{*}(c)-i \sigma(c)$ is analytic, and

$$
\begin{aligned}
\left(\alpha, \sigma^{*}(c)-i \sigma(c)\right) & =\left(\alpha, \sigma^{*}(c)\right)+i(\alpha, \sigma(c)) \\
& =\left(\alpha, \sigma^{*}(c)\right)+i\left(\alpha^{*}, \sigma^{*}(c)\right) \quad\left(\alpha^{*}=-i \alpha\right) \\
& =\left(\alpha, \sigma^{*}(c)\right)+\left(\alpha, \sigma^{*}(c)\right)=2 \int_{c} \alpha \\
& =(\alpha, 2 \psi(c)) .
\end{aligned}
$$

If $c$ and $c^{\prime}$ are cycles, then $\left(\sigma\left(c^{\prime}\right), \sigma^{*}(c)\right)$ is an integer, the actual intersection number, $c^{\prime} \times c$, of $c^{\prime}$ and $c$.

In order to investigate systematically periods on surfaces of infinite genus, we must introduce a canonical homology basis for the nondividing cycles. Ahlfors [1] has shown the following to be possible:

TheOREM 1. Given an exhaustion $\left(^{4}\right)\left\{\Omega_{n}\right\}$ of $W$, there exists a sequence of cycles $A_{1}, B_{1}, A_{2}, B_{2}, \cdots, A_{p(n)}, B_{p(n)}, \cdots$, such that $A_{1}, B_{1}, \cdots, A_{p(n)}, B_{p(n)}$ form a basis modulo the dividing cycles of $\Omega_{n}$, and the cycles have the following intersection properties:

$$
\begin{aligned}
& A_{j} \times A_{k}=B_{j} \times B_{k}=0 \quad \text { for all } j, k, \\
& A_{j} \times B_{k}=\delta_{j k} .
\end{aligned}
$$

From the definition of dividing cycles, it follows that if $c$ is a dividing cycle then $A_{j} \times c=B_{j} \times c=0$ for all $j . \sigma\left(A_{k}\right)$ has zero periods on all cycles of the canonical basis except on $B_{k}$. Similarly for $\sigma\left(B_{k}\right)$.

Note that a canonical homology basis will be referred to as a particular exhaustion.

We quote some further results which we will need later.

THEOREM 2. $\Gamma_{h o}$ is spanned by the $\sigma(c)$ 's as $c$ runs through all cycles.

In order to illustrate techniques used in this paper we include a proof of the following well known theorem.

(4) We will use the notation $\Omega$ with or without subscripts to denote analytically embedded subregions. 
TheOREM 3 (RIEMANN's BILINEAR RELATION). Let $\left\{\Omega_{n}\right\}$ be an exhaustion of $W$ and $A_{1}, B_{1}, \cdots, A_{p(n)}, B_{p(n)}, \cdots$ be a corresponding canonical homology basis. Suppose that $\omega \in \Gamma_{h o}, \sigma \in \Gamma_{h s e}$, and $\sigma$ has a finite number of nonzero $A$ and $B$ periods. Then

$$
\left(\omega, \sigma^{*}\right)=\sum_{k=1}^{\infty} \int_{A_{k}} \omega \int_{B_{k}} \bar{\sigma}-\int_{A_{k}} \bar{\sigma} \int_{B_{k}} \omega, \quad \text { a finite sum. }
$$

Proof. Let $a_{k}=\int_{A_{k}} \sigma$ and $B_{k}=\int_{B_{k}} \sigma$. Let $\sigma^{\prime}=\sum_{k=1}^{\infty} b_{k} \sigma\left(A_{k}\right)-a_{k} \sigma\left(B_{k}\right)$. $\sigma^{\prime}$ has the same periods as $\sigma$, and $\sigma^{\prime} \in \Gamma_{h o} \subset \Gamma_{h s e}$. Then $\sigma-\sigma^{\prime}$ has no periods; that is, $\sigma-\sigma^{\prime} \in \Gamma_{h e}$. Therefore $\left(\omega, \sigma^{*}-\sigma^{*}\right)=0$ since $\Gamma_{h o} \perp \Gamma_{h e}^{*}$. Thus

$$
\left(\omega, \sigma^{*}\right)=\left(\omega, \sigma^{*}\right)=\sum \bar{b}_{k} \int_{A_{k}} \omega-\bar{a}_{k} \int_{B_{k}} \omega . \quad \text { q.e.d. }
$$

Theorem 3 includes the classical result for closed surfaces.

Theorem 4. Suppose $\bar{\Omega}$ is a compact bordered surface and $\omega$ and $\sigma$ are in $\Gamma_{\text {hse }}(\bar{\Omega})$. Suppose further that $A_{1}, B_{1}, \cdots, A_{n}, B_{n}$ is a canonical basis for the cycles of $\Omega$. Then

$$
\left(\omega, \sigma^{*}\right)=\sum_{k=1}^{n} \int_{A_{k}} \omega \int_{B_{k}} \bar{\sigma}-\int_{A_{k}} \bar{\sigma} \int_{B_{k}} \omega+\int_{\partial \Omega} u \bar{\sigma}
$$

where $u$ is a function defined separately on each contour of the boundary, $\partial \Omega$, of $\bar{\Omega}$. If $\alpha$ is a contour of $\partial \Omega$, then $u(p)=\int_{p_{0}}^{p} \omega$ where $p_{0}$ is a fixed point on $\alpha$ and the integration is in the positive sense of $\alpha$.

The proof of Theorem 4 is similar to that of Theorem 3. The correction term $\int_{\partial \Omega} u \bar{\sigma}$ arises from an application of Green's theorem. This correction is necessary since we assume only that $\omega$ is semi-exact.

We now quote some classical results which we seek to generalize in this paper. For a closed surface $W$ the class of analytic differentials is a finite dimensional vector space of dimension equal to the genus $g$ of the surface. If $A_{1}, B_{1}, \cdots, A_{\theta}, B_{\theta}$ is a canonical homology basis for the cycles of $W$, then an analytic differential is determined by its $A$ periods. If $\phi_{k}$ is the unique analytic differential satisfying $\int_{A_{j}} \phi_{k}=\delta_{j k}$ then for any analytic differential $\alpha$ we have $\alpha=\sum_{k=1}^{0} a_{k} \phi_{k}$ where $a_{k}=\int_{A_{k}} \alpha$. These results follow simply from Theorem 3.

The problem of generalizing Theorem 3 is that of showing the convergence of the appropriate infinite sum to the desired inner product. An obvious method is to apply Theorem 4 to differentials in $\Gamma_{h s e}(W)$ restricted to $\Omega_{n}$ where $\left\{\Omega_{n}\right\}$ is an exhaustion of a particular type. The problem then is to show the vanishing of $\int_{\partial \Omega_{n}} u \bar{\sigma}$ as $\Omega_{n} \rightarrow W$.

In 1947 Ahlfors [1] showed for parabolic surfaces that the bilinear relation holds with respect to certain exhaustions if $\omega$ and $\sigma$ both have a finite number 
of nonzero $A$ periods. Some further generalizations of Theorem 3 have been obtained by imposing conditions on the metric structure of the surface. Here Pfluger [1] in the hyperelliptic case and Kusunoki [1] in a more general parabolic situation have obtained results. While the techniques used by these authors have yielded only partial results, the present author has found their approach very useful, especially in constructing examples and counterexamples.

The main result used in this paper for generalizing Theorem 3 is a necessary and sufficient condition for the validity of the bilinear relation with respect to a given canonical homology basis. The sufficiency of this condition for surfaces of class $O_{H D}$ is due to Pfluger [2, pp. 187-189]. The method of proof follows elementary Banach space theory.

An analytic differential $\phi_{k}$ satisfying $\int_{A} \phi_{k}=\delta_{j k}$ will be called a normal differential. Virtanen [1] has established the existence of normal differentials for any surface of class $O_{H D}$. Their unicity, then, is the remaining question. Fortunately, one only needs a weaker form of the bilinear relation in order that an analytic differential on a surface of class $O_{H D}$ be determined by its $A$ periods. In $\S I I$ we give a general criterion for the validity of this weaker form on surfaces of class $O_{H D}$. This criterion, however, is not necessary. \$II includes an explicit construction of normal differentials. We also prove that if an analytic differential is determined by its $A$ periods, then the normal differentials span $\Gamma_{a}$. This latter result is also due to Virtanen [1]. While our proofs are essentially the same as those of Virtanen, they seem to be technically simpler. Finally, we prove that if the most general form of the bilinear relation holds for all harmonic differentials on a surface of class $O_{H D}$, then for $\alpha \in \Gamma_{a}, \sum_{k=1}^{p(n)} a_{k} \phi_{k}$ converges strongly to $\alpha$, where $a_{k}=\int_{A_{k}} \alpha$.

§III is concerned with examples which show that the theory of $\S I I$ is neither vacuous nor all-encompassing. We give a general criterion which insures the validity of the most general form of the bilinear relation. This condition, however, is very strong. The most important counter-example is a parabolic surface where the most general form of the bilinear relation does not hold for the móst natural exhaustion. We also exhibit a surface where $\Gamma_{h m}$ is a proper subset of $\Gamma_{h o} \cap \Gamma_{h e}$.

II. General theory. Let $W$ be a Riemann surface. Let $\left\{\Omega_{n}\right\}$ be an exhaustion of $W$, and let $A_{1}, B_{1}, \cdots, A_{p(n)}, B_{p(n)}, \cdots$ be a corresponding canonical homology basis so that $A_{1}, B_{1}, \cdots, A_{p(n)}, B_{p(n)}$ is a basis modulo the dividing cycles of $\Omega_{n}$.

Definition. For $\sigma \in \Gamma_{h}(W)$ we define $T_{n} \sigma$ as follows:

$$
T_{n} \sigma=\sum_{k=1}^{p(n)} b_{k} \sigma\left(A_{k}\right)-a_{k} \sigma\left(B_{k}\right)
$$

where $a_{k}=\int_{A_{k}} \sigma$ and $b_{k}=\int_{B_{k}} \sigma$. 
Thus $T_{n} \sigma$ is in $\Gamma_{h o}(W)$ and has the same periods as $\sigma$ for $A_{1}, \cdots, B_{p(n)}$, all other $A$ and $B$ periods vanishing.

Definition. For $\sigma \in \Gamma_{h s e}(W)$ we will say that the generalized bilinear relation holds for $\sigma$ if for all $\omega \in \Gamma_{h o}$, we have

$$
\left(\omega, \sigma^{*}\right)=\lim _{n \rightarrow \infty}\left(\omega,\left(T_{n} \sigma\right)^{*}\right)=\lim _{n \rightarrow \infty} \sum_{k=1}^{p(n)} \int_{A_{k}} \omega \int_{B_{k}} \bar{\sigma}-\int_{A_{k}} \bar{\sigma} \int_{B_{k}} \omega .
$$

Theorem 1. Let $\left\{\Omega_{n}\right\}$ and $p(n)$ be as above. Choose $\sigma \in \Gamma_{h s e}$. Let $\left(T_{n} \sigma\right)^{*}$ $=O_{n} \sigma+E_{n} \sigma$ where $O_{n} \sigma \in \Gamma_{h o}$ and $E_{n} \sigma \in \Gamma_{h e}^{*}$. A necessary and sufficient condition that the generalized bilinear relation hold for $\sigma$ is that $\left\|O_{n} \sigma\right\|$ be bounded as $n \rightarrow \infty$.

Remarks. If $W \in O_{H D}$ the sufficiency is due to Pfluger [2]. If $W \in O_{H D}$ then $\left(T_{n} \sigma\right)^{*}$ already lies in $\Gamma_{h o}=\Gamma_{h}$.

Proof. Sufficiency. For any $\omega \in \Gamma_{h o}(W)$ we wish to show that $\left(\omega,\left(T_{n} \sigma\right)^{*}\right)$ $\rightarrow\left(\omega, \sigma^{*}\right)$ as $n \rightarrow \infty$.

Let $O_{n_{j}} \sigma$ be a subsequence of $O_{n} \sigma$ converging weakly to $\tau \in \Gamma_{h o}$. Since $\left\|O_{n} \sigma\right\|$ is bounded there is at least one such subsequence. For all $\omega \in \Gamma_{h o}$ we have

$$
\left(\omega, O_{n_{j}} \sigma\right) \rightarrow(\omega, \tau) \quad \text { as } n \rightarrow \infty .
$$

Since $E_{n_{j}} \sigma$ is orthogonal to $\Gamma_{h o}(W)$ we have further for all $\omega \in \Gamma_{h o},\left(\omega,\left(T_{n_{j}} \sigma\right)^{*}\right)$ $\rightarrow(\omega, \tau)$. Choosing $\omega$ to be $\sigma(c)$ where $c$ is a cycle, we have

$$
\left(\left(T_{n_{j}} \sigma\right)^{*}, \sigma(c)\right) \rightarrow(\tau, \sigma(c))=\int_{c} \tau^{*}
$$

If $c=A_{k}$ or $B_{k},\left(\left(T_{n_{j}} \sigma\right)^{*}, \sigma(c)\right) \rightarrow-\int_{c} \sigma$ by virtue of the definition of $T_{n_{j}} \sigma$. If $c$ is a dividing cycle, $\left(\left(T_{n_{j}} \sigma\right)^{*}, \sigma(c)\right)=0$ for all $j$. Thus $-\sigma$ and $\tau^{*}$ have the same periods. Since $\tau \in \Gamma_{h o}$, this last result uniquely determines $\tau$ independently of the subsequence $n_{j}$. Since $\left\|O_{n} \sigma\right\|$ is bounded we see that $O_{n} \sigma \rightarrow \tau$ weakly since every weakly convergent subsequence does. Thus we have:

$$
\left(\omega,\left(T_{n} \sigma\right)^{*}\right) \rightarrow(\omega, \tau) .
$$

Since $\tau^{*}+\sigma$ is exact we have for all $\omega \in \Gamma_{h o}(W)$

$$
(\omega, \tau)=\left(\omega, \sigma^{*}\right)
$$

which together with equation (1) gives the desired result.

Necessity. If $\left(\omega,\left(T_{n} \sigma\right)^{*}\right) \rightarrow\left(\omega, \sigma^{*}\right)$ for all $\omega \in \Gamma_{h o}$, then $\left(\omega,\left(T_{n} \sigma\right)^{*}\right)$ is bounded for all $\omega \in \Gamma_{h o}$. The result follows from the next theorem.

THEOREM 2. In order that $\left\|O_{n} \sigma\right\|$ be bounded as $n \rightarrow \infty$, it suffices that $\left(\omega,\left(T_{n} \sigma\right)^{*}\right)$ be bounded for each $\omega \in \Gamma_{h o}$, the bound possibly depending on $\omega$.

Proof. Remember that $\left(\omega,\left(T_{n} \sigma\right)^{*}\right)=\left(\omega, O_{n} \sigma\right)$. Since every continuous linear functional on $\Gamma_{h o}$ is expressed by an inner product, the hypothesis 
means that $F\left(O_{n} \sigma\right)$ is bounded for each $F$ in the conjugate space of $\Gamma_{h o}$. This implies that $\left\|O_{n} \sigma\right\|$ is bounded. q.e.d.

The question arises as to when the generalized bilinear relation converges in a uniform manner. The answer is that $O_{n} \sigma$ must approach its weak limit $\tau$ strongly.

Definition. The generalized bilinear expression $\left(\omega,\left(T_{n} \sigma\right)^{*}\right)$ is said to converge uniformly for $\sigma \in \Gamma_{h s e}$ if for all $\epsilon>0$, there is an $n_{0}$, so that for all $\omega \in \Gamma_{h o}$ and $n>n_{0}$ we have

$$
\left|\left(\omega, \sigma^{*}\right)-\left(\omega,\left(T_{n} \sigma\right)^{*}\right)\right|<\epsilon\|\omega\| .
$$

THEOREM 3. The generalized bilinear relation converges uniformly for $\sigma \in \Gamma_{h s e}$ if and only if $O_{n} \sigma$ converges strongly to its weak limit $\tau$.

Proof. We continue the notation of the previous theorems.

Suppose the bilinear relation converges uniformly for $\sigma \in \Gamma_{h s e}$. We know by Theorem 1 that $\left\|O_{n} \sigma\right\|$ is bounded, say by $M$, as $n \rightarrow \infty$. If $\epsilon>0$, we choose $n_{0}$ so that if $n>n_{0}$ we have

$$
\left|\left(\omega, \sigma^{*}-\left(T_{n} \sigma\right)^{*}\right)\right|=\left|\left(\omega, \tau-O_{n} \sigma\right)\right|<\frac{\epsilon\|\omega\|}{\|\tau\|+M} .
$$

If $n>n_{0}$, we let $\omega=\tau-O_{n} \sigma$ and note that $\|\omega\| \leqq\|\tau\|+M$. Thus $\left\|\tau-O_{n} \sigma\right\|^{2}<\epsilon$ for all $n>n_{0}$; that is $O_{n} \sigma \rightarrow \tau$ strongly.

Conversely, if $\left\|\tau-O_{n} \sigma\right\| \rightarrow 0$ as $n \rightarrow \infty$, then

$$
\begin{aligned}
\left|\left(\omega, \sigma^{*}-\left(T_{n} \sigma\right)^{*}\right)\right| & =\left|\left(\omega, \tau-O_{n} \sigma\right)\right| \\
& \leqq\|\omega\|\left\|\tau-O_{n} \sigma\right\|
\end{aligned}
$$

If $W \in O_{H D}$ then the condition of Theorem 3 is that $T_{n} \sigma \rightarrow \sigma$ strongly. In this case we can write $\sigma=\lim _{n} T_{n} \sigma$.

We have considered so far the validity of the generalized bilinear relation for a single differential $\sigma \in \Gamma_{h s e}$. In $\S I I I$ we shall give examples of surfaces which show that the generalized bilinear relation may hold for all differentials of class $\Gamma_{h s e}$ but need not. We continue here with a more general discussion.

If the generalized bilinear relation holds for all harmonic differentials on a surface of class $O_{H D}$, then an analytic differential whose $A$ periods vanish is itself zero. Fortunately, for surfaces of class $O_{H D}$ one needs only the validity of a weaker form of the bilinear relation in order that analytic differentials be determined by their $A$ periods.

Definition. If $W$ is of class $O_{H D},\left\{\Omega_{n}\right\}$ an exhaustion of $W$ and $A_{1}, B_{1}, \cdots, A_{p(n)}, B_{p(n)}, \cdots$ a corresponding canonical homology basis, then we say that the finite bilinear relation holds on $W$, if the bilinear relation holds for $\sigma$ and $\omega$ when they both have a finite number of nonvanishing $A$ periods; that is, 


$$
\left(\omega, \sigma^{*}\right)=\sum_{k=1}^{\infty} \int_{A_{k}} \omega \int_{B_{k}} \bar{\sigma}-\int_{A_{k}} \bar{\sigma} \int_{B_{k}} \omega
$$

a finite sum.

The following lemma establishes some useful conditions which are equivalent to the validity of the finite bilinear relation. We must, however, first introduce two subspaces of $\Gamma_{h}$ that are important.

Definition. $\mathfrak{A}$ is the subspace of $\Gamma_{h}$ spanned by the $\sigma\left(A_{k}\right)$ 's. $\mathfrak{B}$ is the subspace of $\Gamma_{h}$ spanned by the $\sigma\left(B_{k}\right)$ 's.

Thus $\mathfrak{A}$ and $\mathfrak{B}$ depend on the canonical homology basis.

LEMma 4. If $W \in O_{H D}$ then the following three conditions are equivalent:

(a) The finite bilinear relation holds.

(b) $\Gamma_{h}=\mathfrak{A}+\mathfrak{A}^{*}$, an orthogonal decomposition.

(c) The $\psi\left(A_{k}\right)$ 's are complete in $\Gamma_{a}$; that is, if $\alpha \perp \psi\left(A_{k}\right)$ for all $k$, then $\alpha=0$.

Proof. (a) implies (c): Suppose $\alpha \in \Gamma_{a}$ has all its $A$ periods zero. Since $\alpha^{*}=-i \alpha$ it follows that $i(\alpha, \alpha)=\left(\alpha, \alpha^{*}\right)$. Applying the finite bilinear relation to $\left(\alpha, \alpha^{*}\right)$ yields the result.

(c) implies (b): Take a harmonic differential $\omega$ which we assume to begin with is real. If $\omega \perp \mathfrak{A}+\mathfrak{A}^{*}$, then $\omega+i \omega^{*} \perp \mathfrak{A}^{*}$. Thus $\omega+i \omega^{*}$ is analytic and has zero $A$ periods; thus $\omega+i \omega^{*}=0$. Hence $\omega=0$.

If $\omega$ is arbitrary and $\omega \perp \mathfrak{A}+\mathfrak{H}^{*}$, then the real and imaginary parts of $\omega$ are also perpendicular to $\mathfrak{A}+\mathfrak{A}^{*}$.

(b) implies (a): Suppose that $\omega$ and $\sigma$ have a finite number of $A$ periods. Let $w_{k}=\int_{A_{k}} \omega$ and $s_{k}=\int_{A_{k}} \sigma$. Then $\omega+\sum w_{k} \sigma\left(B_{k}\right)$ and $\sigma+\sum s_{k} \sigma\left(B_{k}\right)$ both have no $A$ periods; that is, both are orthogonal to $\mathfrak{A}^{*}$. Since by assumption $\mathfrak{A}$ equals $\mathfrak{X}^{* \perp}$, both are in $\mathfrak{A}$. Thus $\left(\omega+\sum w_{k} \sigma\left(B_{k}\right), \sigma^{*}+\sum s_{k} \sigma^{*}\left(B_{k}\right)\right)=0$. Expanding this yields the desired result. q.e.d.

If $W \in O_{H D}$ then $\mathfrak{A}$ and $\mathfrak{B}$ span $\Gamma_{h}$, but the vector sum $\mathfrak{A}+\mathfrak{B}$ need not be closed as an example of the next section will show. If such a decomposition of $\Gamma_{h}$ holds, we cannot assume that it is orthogonal.

Theorem 5. Suppose $W \in O_{H D}$. If the vector sum $\mathfrak{A}+\mathfrak{B}$ is closed and so equals $\Gamma_{h}$, then the $\psi\left(A_{k}\right)^{\prime}$ s are complete in $\Gamma_{a}$.

Proof. Suppose $\omega$ has zero $A$ periods; that is $\omega \perp \mathscr{A}^{*}$. We will show that $\omega \in \mathfrak{A}$ and apply criterion (b) of Lemma 4.

The hypothesis allows us to write $\omega=a+b$, where $a \in \mathfrak{A}$ and $b \in \mathfrak{B}$. Since $a \perp \mathfrak{H}^{*}$ and $\omega \perp \mathfrak{A}^{*}$, we have $b \perp \mathfrak{A}^{*}$. But $b \perp \mathfrak{B}^{*}$, and so $b$ has no $A$ or $B$ periods. Thus $b=0$ and $\omega \in \mathfrak{A}$. q.e.d.

We now give an explicit construction of the normal differentials. Let $\sigma\left(B_{k}\right)=\theta_{k}+\tau_{k}$ where $\theta_{k} \in \mathfrak{A}^{*}$ and $\tau_{k} \perp \mathfrak{A}^{*}$. Since $\sigma\left(B_{k}\right)$ is real and $\mathfrak{A}^{*}$ is spanned by real differentials, we know that $\theta_{k}$ and $\tau_{k}$ are real differentials. Let $\phi_{k}$ $=-\theta_{\mathfrak{k}}^{*}-i \theta_{\mathfrak{k}}^{*}$, an analytic differential. Since $\theta_{\mathbf{k}}^{*} \perp \mathfrak{A}^{*}$ and $\tau_{k} \perp \mathfrak{A}^{*}$, we have 


$$
\begin{aligned}
\int_{A_{j}} \phi_{k} & =\left(-\theta_{k}-i \theta_{k}^{*}, \sigma^{*}\left(A_{j}\right)\right) \\
& =\left(-\theta_{k}, \sigma^{*}\left(A_{j}\right)\right) \\
& =\left(-\theta_{k}-\tau_{k}, \sigma^{*}\left(A_{j}\right)\right) \\
& =\delta_{k j} .
\end{aligned}
$$

Note that this construction does not depend on $W$ being of class $O_{H D}$.

Lemma 6. If $W \in O_{H D}$ then the $\theta_{k}$ 's span $\mathfrak{A}^{*}$.

Proof. Suppose $\sigma \in \mathfrak{A}^{*}$ and $\sigma \perp \theta_{k}$ for all $k$. Then $\sigma^{*} \in \mathfrak{A}$ and therefore $\sigma^{*} \perp \mathfrak{A}^{*}$; that is, $\sigma^{*}$ has no $A$ periods. Since $\tau_{k} \perp \mathfrak{A}^{*}$ by definition, we have $\sigma \perp \theta_{k}+\tau_{k}$ for all $k$; that is $\sigma \perp \sigma\left(B_{k}\right)$ for all $k$. Thus $\sigma^{*} \perp \sigma^{*}\left(B_{k}\right)$ for all $k$. Therefore $\sigma^{*}$ has no $B$ periods, and so $\sigma^{*}=0$. q.e.d.

We now show that if an analytic differential is determined by its $A$ periods then the normal differentials span $\Gamma_{a}$. This generalizes to an extent the situation for compact surfaces.

Theorem 7 (Virtanen). Suppose $W \in O_{H D}$. If the $\psi\left(A_{k}\right)$ 's span $\Gamma_{a}$, then the $\phi_{k}$ 's also span $\Gamma_{a}$.

Proof. Suppose $\alpha \perp \phi_{k}$ for all $k$. Then $2\left(\alpha, \theta_{k}\right)=\left(\alpha, \theta_{k}\right)+\left(\alpha^{*}, \theta_{k}^{*}\right)=\left(\alpha, \theta_{k}\right)$ $-i\left(\alpha, \theta_{k}^{*}\right)=\left(\alpha, \theta_{k}+i \theta_{k}^{*}\right)=\left(\alpha,-\phi_{k}\right)=0$. By Lemma 6 we have $\alpha \perp \mathfrak{A} *$. Thus $\alpha$ has vanishing $A$ periods, and so $\alpha=0$. q.e.d.

Since all the steps in this proof are reversible we have also proven the converse of Theorem 7 . Thus in Lemma 4 we may add a fourth equivalent condition that the $\phi_{k}$ 's span $\Gamma_{a}$.

We now show that if the generalized bilinear relation holds on $W \in O_{H D}$, then we can express $\alpha$ as the weak limit of $\sum_{\substack{p=1 \\(n)}} a_{k} \phi_{k}$, where $a_{k}=\int_{A_{k}} \alpha$.

THEOREM 8. Suppose that $W \in O_{H D}$. Let $\left\{\Omega_{n}\right\}$ be an exhaustion and $A_{1}, B_{1}, \cdots, A_{p(n)}, B_{p(n)}, \cdots$ a corresponding canonical homology basis with respect to which the finite bilinear relation holds. Suppose further that the generalized bilinear relation holds for $\alpha \in \Gamma_{a}$. If $a_{k}=\int_{A k} \alpha$ and $b_{k}=\int_{B_{k}} \alpha$, then $\sum_{k=1}^{p(n)} a_{k} \phi_{k}$ converges weakly to $\alpha$ as $n \rightarrow \infty$.

Proof. By Theorems 1 and 2 we know that $T_{n} \alpha \in \Gamma_{h o}$ is bounded in norm as $n \rightarrow \infty$.

$$
T_{n} \alpha=\sum_{k=1}^{p(n)} b_{k} \sigma\left(A_{k}\right)-a_{k} \sigma\left(B_{k}\right) .
$$

Let $\hat{\alpha}_{p(n)}$ be the projection of $T_{n} \alpha$ on $\mathfrak{A}^{*}$. Then

$$
\hat{\alpha}_{p(n)}=-\sum_{k=1}^{p(n)} a_{k} \theta_{k}
$$


and $\left\|\hat{\alpha}_{p(n)}\right\|$ is bounded as $n \rightarrow \infty$. Then

$$
\hat{\alpha}_{p(n)}+i \hat{\alpha}_{p(n)}^{*}=-\sum_{k=1}^{p(n)} a_{k}\left(\theta_{k}+i \theta_{k}^{*}\right)=\sum_{k=1}^{p(n)} a_{k} \phi_{k}
$$

is bounded in norm as $n \rightarrow \infty$. Since analytic differentials are determined by their $A$ periods, we see by checking periods that every weakly convergent subsequence of $\sum_{k=1}^{p(n)} a_{k} \phi_{k}$ converges to $\alpha$. Therefore, $\sum_{k=1}^{p(n)} a_{k} \phi_{k} \rightarrow \alpha$ weakly. q.e.d.

Note that we may write the results of the last theorem as follows: $\sum_{k=1}^{p(n)}\left(\alpha, \psi\left(A_{k}\right)\right) \phi_{k} \rightarrow \alpha$ weakly. Suppose the generalized bilinear relation holds for all harmonic differentials on $W$. Then $\sum^{p(n)}\left(\alpha, \psi\left(A_{k}\right)\right)\left(\phi_{k}, \beta\right)$ $\rightarrow(\alpha, \beta)$ for all $\alpha, \beta \in \Gamma_{a}$. Consequently $\sum^{p(n)}\left(\beta, \phi_{k}\right) \psi\left(A_{k}\right) \rightarrow \beta$ weakly. We have thus proved the following corollary.

COROLLARY 9. If the generalized bilinear relation holds for all $\omega \in \Gamma_{h}$ on $W \in O_{H D}$, then for $\alpha \in \Gamma_{a}$ we have

$$
\sum_{k=1}^{p(n)}\left(\alpha, \phi_{k}\right) \psi\left(A_{k}\right) \rightarrow \alpha \text { weakly. }
$$

If $W \in O_{H D}$ then the $\sigma(A)$ 's and the $\sigma(B)$ 's span $\Gamma_{h}$. If the generalized bilinear relation holds then the $\phi_{k}$ 's span $\Gamma_{a}$. This additional information allows us to apply a theorem of Banach-Karlin [1] to assert that all weak convergences are, in fact, strong convergences.

THEOREM 10. If the generalized bilinear relation holds for all harmonic differentials on $W \in O_{H D}$ then

$$
\begin{aligned}
T_{n} \sigma & \rightarrow \sigma \text { strongly for } \sigma \in \Gamma_{h}, \\
\sum\left(\alpha, \psi_{k}\right) \phi_{k} & \rightarrow \alpha \text { strongly for } \alpha \in \Gamma_{a},
\end{aligned}
$$

and

$$
\sum\left(\alpha, \phi_{k}\right) \psi_{k} \rightarrow \alpha \text { strongly. }
$$

This follows from the fact that $\lim _{n} T_{n} \sigma(c)=\sigma(c)$ for all cycles $c$ and each $\sigma \in \Gamma_{h}$ may be approximated arbitrarily closely be finite linear combinations of the $\sigma(A)$ 's and $\sigma(B)$ 's. Analogous reasoning proves the corresponding result for the analytic differentials.

Since $\left\{T_{n}\right\}$ is a sequence of linear operators on $\Gamma_{h}$ we may interpret the above result as follows:

COROLLARY 11. The generalized bilinear relation holds for all harmonic differentials on $W \in O_{H D}$ if and only if the norms $\left\|T_{n}\right\|$ are bounded as $n \rightarrow \infty$.

We may summarize the above results by saying that the validity of the generalized bilinear relation on a surface of class $O_{H D}$ insures us of the results 
which we obtain in the classical situation by means of the classical Riemann bilinear relation.

We conclude this section with a theorem which connects another form of the bilinear relation with the class of harmonic measures.

Definition. The special bilinear relation will be said to hold on a surface if the following is true: if $\omega \in \Gamma_{h o}, \sigma \in \Gamma_{h s e}$ and $\omega$ has a finite number of nonzero $A$ and $B$ periods, then

$$
\left(\omega, \sigma^{*}\right)=\sum_{k=1}^{\infty} \int_{A_{k}} \omega \int_{B_{k}} \bar{\sigma}-\int_{A_{k}} \bar{\sigma} \int_{B_{k}} \omega, \quad \text { a finite sum. }
$$

THEOREM 12. The validity of the special bilinear relation on a surface $W$ is equivalent to $\Gamma_{h m}(W)=\Gamma_{h o}(W) \cap \Gamma_{h e}(W)$.

Proof. If the special bilinear relation holds and $\omega \in \Gamma_{h o} \cap \Gamma_{h e}$, then $\omega$ has no $A$ or $B$ periods. Thus $\left(\omega, \sigma^{*}\right)=0$ for all $\sigma \in \Gamma_{h s e}$ and so $\omega \in \Gamma_{h m}(W)$. Note that $\Gamma_{h m} \subset \Gamma_{h o} \cap \Gamma_{h e}$ always.

Conversely, if $\omega \in \Gamma_{h o}$ and $a_{k}=\int_{A_{k}} \omega$ and $b_{k}=\int_{B_{k}} \omega$ then $\omega^{\prime}=\omega-\sum_{k=1}^{\infty} b_{k} \sigma\left(A_{k}\right)$ $-a_{k} \sigma\left(B_{k}\right)$ is in $\Gamma_{h o} \cap \Gamma_{h e}$. Thus $\omega^{\prime}$ is in $\Gamma_{h m}$ and so $\left(\omega^{\prime}, \sigma^{*}\right)=0$. Expanding $\left(\omega^{\prime}, \sigma^{*}\right)$ yields the result.

III. Examples and counter examples. To construct surfaces which illustrate the previous theory we must impose strong conditions on the metric structure of the surface. The first lemma will establish our chief tool. Before proceeding to the lemma we will define for each finite surface $\Omega$ a constant $\mu_{\Omega}$ that measures the "width of the edge" of $\Omega$.

Let $\Omega$ be a finite Riemann surface. Suppose $\partial \Omega=\cup_{i=1}^{n} \Gamma_{i}$ where the $\Gamma_{i}$ are the individual contours of $\partial \Omega$. We choose ring domains $R_{i}$ in $\Omega$ so that $\Gamma_{i} \subset \bar{R}_{i}$. We assume that the $R_{i}$ are mutually disjoint. Let $\alpha_{i}$ be the other contour of $R_{i}$ and assume that $\alpha_{i}$ is an analytic Jordan curve. Let $R=\bigcup_{i} R_{i}, \alpha=\bigcup_{i} \alpha_{i}$. Let $g_{R}$ be the harmonic measure of $\alpha$ with respect to $R$. Let $D\left(g_{R}\right)$ be the square of the Dirichlet norm of $d g_{R}$ taken over $R$.

We now consider all possible choices of the $R_{i}$ satisfying the conditions of the last paragraph. Define $\mu_{\Omega}$ to be the infimum of the $D\left(g_{R}\right)$ 's as $R$ ranges over all possible choices.

Lemma 1. Let $\Omega$ be a finite Riemann surface of genus p. Let $\sigma$ and $\omega$ be harmonic on $\bar{\Omega}$ and semi-exact. Let $A_{1}, B_{1}, \cdots, A_{p}, B_{p}$ be a canonical homology basis of $\Omega$. If $a_{k}=\int_{A_{k}} \sigma$ and $b_{k}=\int_{B_{k}} \sigma$ then let $\sigma_{1}=\sum_{k=1}^{p} b_{k} \sigma\left(A_{k}\right)-a_{k} \sigma\left(B_{k}\right)$. Then

$$
\left|\left(\omega, \sigma_{1}^{*}\right)\right| \leqq\|\omega\|\|\sigma\|\left(1+\mu_{\Omega}\right) .
$$

Proof. Choose $\epsilon>0$. Choose $R=\cup_{i} R_{i}$ so that $D\left(g_{R}\right) \leqq \mu_{\Omega}+\epsilon$. Consider the subsurface of $\Omega, \Omega-R=S_{1}$, which has $\alpha$ as its boundary. Since $\alpha$ and $\partial \Omega$ bound ring domains and the components of $\alpha$ are analytic Jordan curves we may choose the homology basis for $S_{1}$ and $\Omega$ to be the same. Now let $S_{t}$ be 
the subsurface bounded by the level curves, $g_{R}=t$. Again $S_{t}$ and $\Omega$ have the same homology basis.

By Theorem 4 of the Introduction we compute $\left(\omega, \sigma^{*}\right)_{s_{t}}\left({ }^{5}\right)$.

(1) $\quad\left(\omega, \sigma^{*}\right)_{S_{t}}=\sum_{k=1}^{p} \int_{A_{k}} \omega \int_{B_{k}} \bar{\sigma}-\int_{A_{k}} \bar{\sigma} \int_{B_{k}} \omega+\int_{\partial S_{t}} u \bar{\sigma}=\left(\omega, \sigma_{1}^{*}\right)+\int_{\partial S_{t}} u \bar{\sigma}$.

$u$ is a function defined separately on each contour, $s_{t}^{t}$, of $S_{t}$ by $u(p)$ $=\int_{p_{0}}^{p} \omega$ where $p_{0} \in s_{t}^{i}$, and integration is in the positive direction along $s_{t}^{t}$.

If $s_{t}^{t}$ is the component of $\partial S_{t}$ lying in $R_{i}$, equation (1) yields

$$
\left|\left(\omega, \sigma_{1}^{*}\right)\right| \leqq\left|\left(\omega, \sigma^{*}\right)_{s_{t}}\right|+\left|\sum_{i} \int_{s_{t}^{i}} u \bar{\sigma}\right| .
$$

We are led by the definition of $u$ to the inequality

$$
\left|\sum_{i} \int_{i_{i}^{i}} u \bar{\sigma}\right| \leqq \sum_{i} \int_{i_{i}^{i}}|\omega| \int_{s_{i}^{i}}|\sigma| \text {. }
$$

Let $h_{R}$ be the conjugate function of $g_{R}$. For convenience we drop the subscript $R$. We will use the notation $\rho_{\omega}$ for $\left(|a|^{2}+|b|^{2}\right)^{1 / 2}$ if $\omega$ locally has the representation $\omega=a d x+b d y$. Using $g+i h$ as a local uniformizer we have

$$
\left|\sum_{i} \int_{i_{i}^{i}} u \bar{\sigma}\right| \leqq \sum_{i} \int_{i_{i}^{i}} \rho_{\omega} d h \int_{i_{i}^{i}} \rho_{\sigma} d h \text {. }
$$

An application of the Schwarz inequality yields

$$
\int_{s_{i}^{i}} \rho_{\omega} d h \leqq\left(\int_{s_{i}^{i}} \rho_{\omega}^{2} d h\right)^{1 / 2}\left(\int_{s_{t}^{i}} d h\right)^{1 / 2} .
$$

If $g^{i}$ is $g$ restricted to $R_{i}$ then $\int_{d}^{i} d h=D\left(g^{i}\right)$ which is less than $D(g)$. Thus

$$
\begin{aligned}
& \left|\sum_{i} \int_{s_{i}^{i}} u \bar{\sigma}\right| \leqq \sum_{i} D\left(g^{i}\right)\left(\int_{s_{i}^{i}} \rho_{\omega}^{2} d h \int_{s_{t}^{i}} \rho_{\sigma}^{2} d h\right)^{1 / 2} \\
& \leqq D(g)\left(\sum_{i} \int_{i_{i} \rho_{\omega}} \rho_{\omega}^{2} d h\right)^{1 / 2}\left(\sum_{i} \int_{i_{i}^{i}} \rho_{\sigma}^{2} d h\right)^{1 / 2}
\end{aligned}
$$

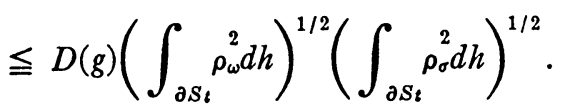

Using inequality (5) we may rewrite inequality (2) as

$$
\left(\omega, \sigma_{1}^{*}\right) \leqq\|\omega\|\|\sigma\|+D(g)\left(\int_{\partial S_{t}} \rho_{\omega}^{2} d h \int_{\partial S_{t}} \rho_{\sigma}^{2} d h\right)^{1 / 2} .
$$

(5) A subscript on an inner product will indicate the region over which the integral is computed. 
Integrating inequality (6) between $g=0$ and $g=1$ and again applying the Schwarz inequality yields

$$
\left|\left(\omega, \sigma_{1}^{*}\right)\right| \leqq\|\omega\|\|\sigma\|+D(g)\left(\int_{g=0}^{1} \int_{\partial S t} \rho_{\omega}^{2} d h d g\right)^{1 / 2}\left(\int_{g=0}^{1} \int_{\partial S t} \rho_{\sigma}^{2} d h d g\right)^{1 / 2} .
$$

Remembering the bound on $D(g)$ we have

$$
\left|\left(\omega, \sigma_{1}^{*}\right)\right| \leqq\|\omega\|\|\sigma\|+\left(\mu_{\Omega}+\epsilon\right)\|\omega\|\|\sigma\| .
$$

Since $\epsilon$ was arbitrary we have the desired result.

If we define $T \sigma=\sigma_{1}$, the above lemma gives a bound on the projection of $T \sigma \in \Gamma_{h o}(\Omega)$ on $\Gamma_{h o} \cap \Gamma_{h s e}^{*}(\Omega)$. Note that the lemma holds if $\omega$ and $\sigma$ are in $\Gamma_{h s e}(\Omega)$. The restriction in the hypothesis eliminates an obvious approximation argument.

We now define a type of finite subsurface which will occur often in this section.

Definition. A finite subsurface $\Omega$ will be called regular if each component of $W-\Omega$ is not relatively compact in $W$ and has only one contour in common with $\Omega$.

THEOREM 2. Let $\left\{\Omega_{n}\right\}$ be an exhaustion of $W$ such that each $\Omega_{n}$ is regular and such that $\mu_{\Omega_{n}}$ is bounded as $n \rightarrow \infty$. Then the generalized bilinear relation holds on $W$ with respect to any canonical homology basis corresponding to the exhaustion $\left\{\Omega_{n}\right\}$.

Proof. In the light of Theorems 1 and 2 of $\S I$ we need only show that if $\omega$ and $\sigma$ are semi-exact on $W$ then $\left(\omega,\left(T_{n} \sigma\right)^{*}\right)$ is bounded as $n \rightarrow \infty$, where $T_{n}$ is defined as before. But since $\omega$ and $\sigma$ are semi-exact on each $\bar{\Omega}_{n}$ we may apply Lemma 1 to conclude that $\left(\omega,\left(T_{n} \sigma\right)^{*}\right) \leqq\left(1+\mu_{\Omega_{n}}\right)\|\omega\|\|\sigma\|$, which by hypothesis is a bound independent of $n$. q.e.d.

Note that if the ring domains corresponding to the $\Omega_{n}$ 's of the exhaustion are disjoint then the surface is parabolic. Note further that the proof does not require the $\Omega_{n}$ to be relatively compact in $W$. Finally it should be remarked that we know of no surface satisfying the hypothesis of Theorem 2 which cannot be imbedded in a parabolic surface.

We now use Theorem 2 to construct a Riemann surface for which the generalized bilinear relation holds. Our example is the symmetric hyperelliptic Riemann surface. Let $\left\{a_{k}\right\}_{k=1}^{\infty}$ be a strictly increasing sequence of positive numbers such that $a_{k} \rightarrow \infty$ as $k \rightarrow \infty$. Denote by $\alpha_{n}$ the segment between $a_{2 n-1}$ and $a_{2 n}$. Now cut the plane along each slit. Take two copies of the slit plane $\pi_{+}$and $\pi_{-}$and cross identify along the slits in the usual way. This gives a two-sheeted ramified covering surface of the finite plane with branch points of multiplicity two over each $a_{n}$. The surface is of infinite genus and parabolic. We exhaust $W$ by the portion $\Omega_{n}$ lying over the open disk with center at zero and radius $a_{2 n} . \Omega_{n}$ is a regular subsurface. Let $R_{n}$ be the ring 
domain in $W$ lying above the annulus $a_{2 n-1}<|z|<a_{2 n}$. Then $D\left(g_{R_{n}}\right)$ is $4 \pi / \log \left(a_{2 n} / a_{2 n-1}\right)$. While $\partial R_{n}$ is not a Jordan curve it is obvious that $\mu_{\Omega_{n}}$ $\leqq D\left(g_{R_{n}}\right)$. In order that $\lim \inf _{n} D\left(g_{R_{n}}\right)<\infty$ we need that $a_{2 n} / a_{2 n-1}>\rho>1$ for a subsequence of the $a_{n}$ 's. This assures, via Theorem 2 , the validity of the generalized bilinear relation for the appropriate exhaustion.

It should be pointed out that the metric conditions imposed on these hyperelliptic surfaces are very strong. If we let $E_{n} \sigma=T_{n} \sigma-T_{n-1} \sigma, T_{0} \sigma=0$, then it can be shown that $\left\|\sum_{k \in A} E_{k} \sigma\right\| \leqq M_{1}\|\sigma\|$ where $A$ is any finite subset of the integers and $M_{1}$ is a constant independent of $A$. It seems improbable that such a strong condition should hold in general on a surface for which the generalized bilinear relation holds; however, we know of no example which does not satisfy the strong condition.

The next part of this section is devoted to surfaces of class $O_{H D}$ for which $\mathfrak{A}$ is orthogonal to $\mathfrak{B}$. We recall that $\mathfrak{A}$ is the subspace of $\Gamma_{h}$ spanned by the $\sigma\left(A_{k}\right)$ 's and $\mathscr{B}$ is the subspace spanned by the $\sigma\left(B_{k}\right)$ 's. For such surfaces we know $\Gamma_{h}=\mathfrak{A}+\mathfrak{B}$, and we may apply Theorem 5 of $\S I$ and its consequences to such surfaces. The surfaces considered will be aribtrary symmetric hyperelliptic surfaces. We will make use of the symmetries these surfaces possess.

We say that a Riemann surface $W$ has an involutory mapping $j$, if $j$ is an anti-conformal map of $W$ onto itself of period two. Corresponding to every differential $\sigma$ on $W$ we form another differential $\tilde{\sigma}$ as follows: if $\sigma=a(z) d x$ $+b(z) d y$ near $\zeta$, then $\tilde{\sigma}=a(j(z)) d x-b(j(z)) d y$ near $j(\zeta)$. We note the following relations:

$$
\begin{aligned}
\int_{c} \sigma & =\int_{j(c)} \tilde{\sigma} \quad \text { for any cycle } c, \\
(\omega, \sigma) & =(\tilde{\omega}, \tilde{\sigma}) .
\end{aligned}
$$

From these relations it follows that $\sigma(j(c))=-\tilde{\sigma}(c)$. For $\left(\omega, \sigma^{*}(c)\right)=\int_{c} \omega$ $=\int_{j(c) \tilde{\omega}}=\left(\tilde{\omega}, \sigma^{*}(j(c)),=\left(\omega,\left[\sigma^{*}(j(c))\right]^{\sim}\right)=\left(\omega,[-\tilde{\sigma}(j(c))]^{*}\right)\right.$ since $(\tilde{\sigma})^{*}$ $=\left(-\sigma^{*}\right) \sim$ and $(\tilde{\omega}) \sim \mathcal{}=$

We say that $\sigma$ is symmetric if $\tilde{\sigma}=\sigma$, and anti-symmetric if $\tilde{\sigma}=-\sigma$. It follows that if $c$ is homologous to $j(c)$ then $\sigma(c)$ is anti-symmetric and if $c$ is homologous to $-j(c)$ then $\sigma(c)$ is symmetric.

If $\sigma$ is a differential, then $\sigma=\sigma_{s}+\sigma_{a}$ where $\sigma_{s}=(\sigma+\tilde{\sigma}) / 2$ and $\sigma_{a}=(\sigma-\tilde{\sigma}) / 2$. Such a decomposition is unique since symmetric and anti-symmetric differentials are perpendicular.

We now define an involutory mapping of the symmetric hyperelliptic Riemann surface onto itself as follows: if $z \in \pi_{+}$then $j(z)$ is $\bar{z}$ of $\pi_{-}$and if $z \in \pi_{-}$then $j(z)$ is $\bar{z}$ of $\pi_{+}$.

In the following diagram we exhibit an explicit canonical homology basis for $W$. The plane of the page will be $\pi_{+}$, and any part of a curve that is dashed will be considered to be in $\pi_{-}$. 


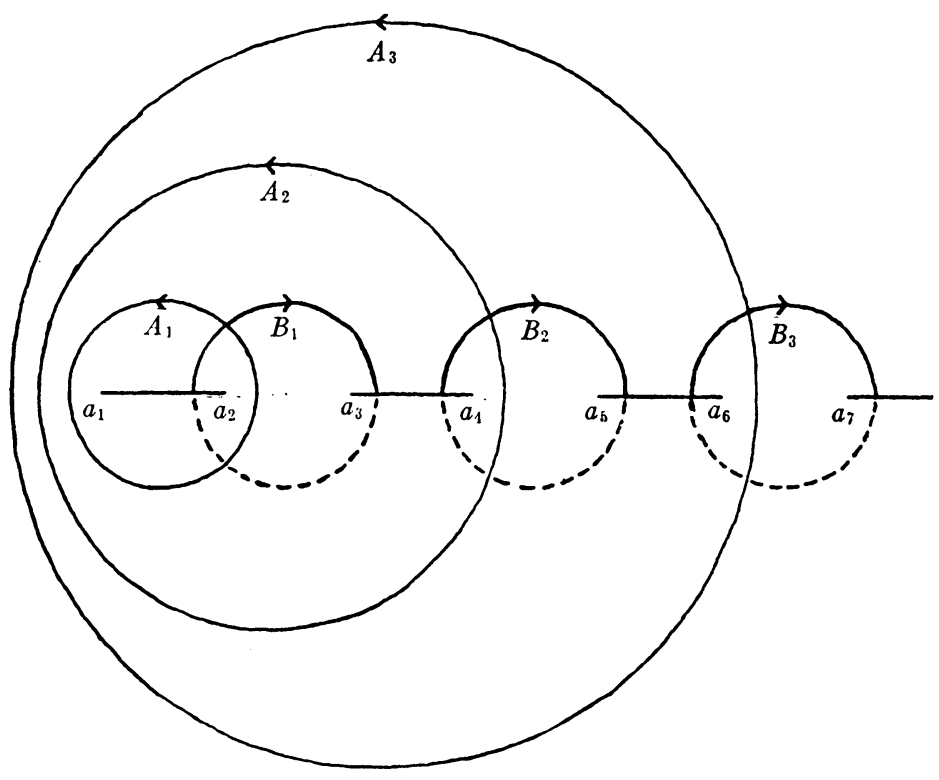

With the exhibited homology basis we see that $j\left(A_{k}\right)$ is homologous to $A_{k}$ and $j\left(B_{k}\right)$ is homologous to $-B_{k}$. Thus $\sigma\left(A_{k}\right)$ is anti-symmetric and $\sigma\left(B_{k}\right)$ is symmetric. Hence $\mathfrak{A} \perp \mathfrak{B}$ for this surface; therefore, we may apply Theorem 5 of $\S 1 \mathrm{I}$.

In these examples the validity of the finite bilinear relation is not a new result since the surfaces are parabolic and the homology bases are of the type considered by Ahlfors [1]. However, we can assert for these symmetric surfaces that an analytic differential is determined by its $B$ periods as well as its $A$ periods. This follows by an obvious symmetric argument.

Our next example shows that the results of Lemma 4 of the last section cannot hold in general. The surface will be a slit torus. Since this surface is of finite genus all forms of the bilinear relation are valid. We will exhibit two distinct analytic Schottky differentials which have an $A$ period of one.

To obtain the torus we identify the inner and outer edge of the annulus $1 \leqq|z| \leqq 4$ by the map $z \rightarrow 4 z$. We excise from the annulus the arc $|z|=2$, $|\theta| \leqq \pi / 4$. Our slit torus thus has one boundary contour; consequently, all closed differentials are semi-exact. For a homology basis the circle $|z|=3$ with the counter-clockwise orientation will be the $A$ cycle, and the line segment starting at -1 and ending at -4 will be the $B$ cycle. The anti-conformal map $z \rightarrow 4 / \bar{z}$ induces an anti-conformal selfmapping $j$ of the slit torus with period two. Since $j(A)$ is homologous to $A$ and $j(B)=-B$, it follows that $\sigma(A)$ is orthogonal to $\sigma(B)$.

With these preliminaries we construct the two normal differentials. Let 
$\phi_{1}$ be the normal differential as constructed in $\S I I$; that is, if $\sigma(B)=\theta+\tau$ where $\theta=\alpha \sigma^{*}(A), \tau \perp \sigma^{*}(A)$, and $\alpha$ is real, then $\phi_{1}=-\theta-i \theta^{*}$. Then $\int_{A} \phi_{1}$ $=\left(\phi_{1}, \sigma^{*}(A)\right)=1$. Let $\phi_{2}=-\sigma(B)-i \sigma^{*}(B)$. Then $\left(\phi_{2}, \sigma^{*}(A)\right)=\left(-\sigma(B), \sigma^{*}(A)\right)$ $=1$. If $\phi_{1}=\phi_{2}$ then $\theta=\sigma(B)$ since $\theta$ and $\sigma(B)$ are real differentials. But $\theta=\alpha \sigma^{*}(A)=\sigma(B)$ contradicts the fact that for this surface $\Gamma_{h o} \cap \Gamma_{h o}^{*}=0$. Thus $\phi_{1} \neq \phi_{2}$. Both $\phi_{1}$ and $\phi_{2}$ are in $\Gamma_{h o}+\Gamma_{h o}^{*}$ and thus are Schottky differentials.

Before proceeding to our next example, we prove a general result which will be necessary.

LEMMa 3. Let $\bar{W}$ be a bordered surface, not necessarily finite. Let $W$ be the interior of $\bar{W}$ and $B$ be the border. If $\omega \in \Gamma_{h o}(W)$ then $\omega$ can be extended to be harmonic on $\bar{W}$ and the extended $\omega$ is zero along $B$.

Proof. Let $\hat{W}$ be the double of $\bar{W}$. Extend the definition of $\omega$ to $\hat{W}-\bar{W}$ so that $\omega$ is anti-symmetric on $W$. Call the extension $\tilde{\omega}$. Choose $P \in B$ and $\Delta \mathrm{A}$ parametric disk in $\hat{W}$ centered at $P$. If we show that $\tilde{\omega}$ and $\tilde{\omega}^{*}$ are orthogonal to all exact differentials of class $C^{1}$ vanishing outside $\Delta$, Weyl's lemma assures us that $\tilde{\omega}$ can be defined at $P$ to be harmonic. The proof of this orthogonality is essentially the same proof as in Ahlfors [3, pp. 11-14]. Assuming that $\tilde{\omega}$ can be extended to be harmonic on all of $W$, it follows that it is zero along $B$ since it is anti-symmetric.

THEOREM 4. Let $\bar{W}$ be a bordered surface, $B$ the boundary and $W$ the interior of $\bar{W}$. Suppose that the double, $\hat{W}$, of $\bar{W}$ is of class $O_{H D}$. Then any square integrable harmonic differential on $W$, defined on $\bar{W}$ and vanishing along $B$ is of class $\Gamma_{h o}(W)$.

Proof. Suppose $\omega \in \Gamma_{h}(W)$ is defined on $\bar{W}$ and vanishes along $B$. Then $\omega=\sigma+\tau^{*}, \sigma \in \Gamma_{h o}(W), \tau \in \Gamma_{h e}(W)$. We show that $\tau=0$.

We may extend $\omega$ by reflection to be anti-symmetric on $\hat{W}$. Call the extension $\varpi$. Since $\sigma$ vanishes along $B$ by Lemma 3, we extend it similarly to be $\tilde{\sigma} . \tau^{*}$ also vanished along $B$ so we extend it to $\tilde{\tau}^{*}$ which is also anti-symmetric. Thus $\tau$ is extended to $\tilde{\tau}$ which is symmetric on $\hat{W}$. We now show $\tilde{\tau}$ is exact and therefore zero since $\hat{W} \in O_{H D}$.

Since $\tau$ is exact on $W, \int_{c} \tilde{\tau}=0$ for any cycle $c$ homologous to a cycle contained entirely in $\bar{W}$ or $\hat{W}-W$. If $c$ is not such a cycle, part of it lies in $\bar{W}$ and part in $\hat{W}-\bar{W}$. Let $c_{1}=c \cap \bar{W}$, and $c_{2}=c \cap \hat{W}-\bar{W}$. Let $c_{1}^{\prime}$ be the reflection of $c_{1}, c_{1}^{\prime}$ lies in $\hat{W}-W$. Then $c=c_{1}-c_{1}^{\prime}+c_{2}+c_{1}^{\prime}$. Therefore $\int_{c} \tilde{\tau}=\int_{c_{1}-c_{1}^{\prime}} \tilde{\tau}$, since $c_{2}+c_{1}^{\prime}$ lies entirely in $\hat{W}-W$. But if $j$ is the reflection of $\hat{W}$ in $B$, then $j\left(c_{1}-c_{1}^{\prime}\right)=c_{1}^{\prime}-c_{1}$, and so $\sigma\left(c_{1}-c_{1}^{\prime}\right)$ is symmetric. Thus $\int_{c} \tilde{\tau}=\left(\tilde{\tau}, \sigma^{*}\left(c_{1}-c_{1}^{\prime}\right)\right)$ $=0$ and $\tilde{\tau}$ is symmetric. $\tilde{\tau}$ vanishes on all cycles of $\hat{W}$; thus $\tilde{\tau}=0$ and so $\tau=0$.

Theorem 4 together with Lemma 3 generalizes to arbitrary bordered surfaces whose double is of class $O_{H D}$ the result for finite surfaces $W$ that states that $\Gamma_{h o}(\bar{W})=\Gamma_{h o}(W)$.

Our next example is a surface for which $\Gamma_{h m}$ is strictly less than $\Gamma_{h o} \cap \Gamma_{h e}$. Since the equality of these two spaces is equivalent to the validity of the 
special bilinear relation and since the generalized bilinear relation trivially implies the special bilinear relation, this example will give us a surface for which the generalized bilinear relation can hold for no exhaustion. On our surface $\Gamma_{h m}=0$. We will exhibit a nonzero differential of class $\Gamma_{h o} \cap \Gamma_{h e}$. Thus our surface will not be of class $O_{H D}$. The question of whether there are surfaces of class $O_{H D}$ for which the generalized bilinear relation does not hold will be answered by our final example.

We start with the interior of the unit circle and an increasing sequence of positive numbers $a_{n}$ such that $a_{n} \rightarrow 1$ as $n \rightarrow \infty$. We choose $b_{n}$ so that $a_{n}<b_{n}<a_{n+1}$, and let $\alpha_{n}$ denote the segment $\left[a_{n}, b_{n}\right]$. We further require that the $b_{n}$ 's be chosen inductively so that the harmonic function $u$ which is one on $|z|=1$ and zero on all the $\alpha_{n}$ has finite Dirichlet norm. Excise from the open unit disk the slits $\alpha_{n}$ and call the resulting slit unit disk $U_{1}$. Let $C_{1}$ denote the circle $|z|=1$ minus the point 1 . To obtain our surface $W$, we double $U_{1}$ simultaneously across all the boundary contours except $C_{1}$; that is, we take an abstract reflection, $U_{2}$, of $U_{1}$ and "glue" it to $U_{1}$ along the slits, $\alpha_{n}$. Let $C_{2}$ be the reflection of $C_{1} . C_{1}$ and $C_{2}$ are infinite boundary contours of $W$. Note that $W$ has no dividing cycles; that is, $\Gamma_{h m}(W)=0$. The function $u$ is extended by reflection so that it is one on $C_{1}$ and minus one on $C_{2}$. Thus $d u$ is of class $\Gamma_{h e}(W)$. Also $d u$ is zero along $C_{1}$ and $C_{2}$. We conclude by Theorem 4 that $d u$ is of class $\Gamma_{h o}(W)$ by observing that if we double $W$ across $C_{1}$ and $C_{2}$ the resulting surface is parabolic.

Our final example exhibits a surface for which the generalized bilinear relation does not hold for the most natural exhaustion. Our surface $W$ is a symmetric transcendental hyperelliptic surface where the slits $\alpha_{n}$ are the intervals $[2 n-1,2 n]$. With the canonical homology basis as depicted before we shall show:

(1) $\left\|\sigma\left(A_{n}\right)-\sigma\left(A_{n-1}\right)\right\|$ is bounded as $n \rightarrow \infty$.

(2) $\left\|\sigma\left(A_{n}\right)\right\| \rightarrow \infty$ as $n \rightarrow \infty$.

Since $T_{n-1}\left(\sigma\left(A_{n}\right)-\sigma\left(A_{n-1}\right)\right)=-\sigma\left(A_{n-1}\right)$ this will suffice to show that $\left\|T_{n}\right\|$ $\rightarrow \infty$. By Corollary 11 of $\S I$ this will show that the generalized bilinear relation holds for no exhaustion for which the given homology basis is a canonical basis.

Let us define the cycles $\left\{C_{n}\right\}$ by $C_{1}=A_{1}$ and $C_{n}=A_{n}-A_{n-1} . C_{n}$ is thus the cycle which "circles" the slit $\alpha_{n}$. We show first that $\left\|\sigma\left(C_{n}\right)\right\|$ is bounded as $n \rightarrow \infty$. Let $R_{n}$ be the annulus in $\pi_{+} 1 / 2 \leqq|z-(2 n-1 / 2)| \leqq 3 / 2$, an annulus in $W$. Let $g_{n}$ be the harmonic function which is zero on $|z-(2 n-1 / 2)|=1 / 2$ and one on $|z-(2 n-1 / 2)|=3 / 2$. Extend the definition of $d g_{n}$ to be zero elsewhere on $W$. Then $d g_{n}$ is a closed differential on $W$. For $\sigma \in \Gamma_{h}(W)\left(\sigma, d g_{n}^{*}\right)$ $=-\iint_{R_{n}} \sigma \wedge d g_{n}=\int_{\partial R_{n}} g_{n} \sigma=\int_{C_{n}} \sigma=\left(\sigma, \sigma^{*}\left(C_{n}\right)\right)$. Since $\sigma\left(C_{n}\right)$ is the closed reproducing differential with minimum norm we have $\left\|\sigma\left(C_{n}\right)\right\|^{2} \leqq\left\|d g_{n}\right\|^{2}$ $=2 \pi / \log 3$.

For the second part we make a similar estimate. Let $D_{p}^{n}=\sum_{k=p}^{2 n-p} B_{k}$. Thus $\left(\sigma\left(A_{n}\right), \sigma^{*}\left(D_{p}^{n}\right)\right)=1$ for $p=1,2, \cdots, n$. Let $G_{p}^{n}$ be the annulus in $W$ : 


$$
\begin{array}{ll}
2 n-2 p+\frac{1}{2} \leqq\left|z-\left(2 n+\frac{1}{2}\right)\right| \leqq 2 n-2 p+\frac{3}{2}, & y \geqq 0 \text { in } \pi_{+}, \\
2 n-2 p+\frac{1}{2} \leqq\left|z-\left(2 n+\frac{1}{2}\right)\right| \leqq 2 n-2 p+\frac{3}{2}, & y \leqq 0 \text { in } \pi_{-}, \\
& p=1, \cdots, n .
\end{array}
$$

Let $\omega_{p}^{n}$ be the harmonic function in $G_{p}^{n}$ such that $\omega_{p}^{n}=0$ on $|z-(2 n+1 / 2)|$ $=2 n-2 p+1 / 2$, and $\omega_{p}^{n}=1$ on $|z-(2 n+1 / 2)|=2 n-2 p+3 / 2$. As before we have for $\sigma$, harmonic in $W$ :

$$
\int_{D_{p}^{n}} \sigma=\left(\sigma, d \omega_{p}^{n *}\right)
$$

Thus

$$
\begin{aligned}
1 & =\left(\sigma\left(A_{n}\right), d \omega_{p}^{n *}\right)^{2} \leqq\left\|\sigma\left(A_{n}\right)\right\|_{G_{p^{n}}}^{2}\left\|d \omega_{p}^{n}\right\|_{G_{p^{n}}}^{2} \\
& \leqq\left\|\sigma\left(A_{n}\right)\right\|_{G_{p^{n}}}^{2} \cdot 2 \pi\left(\log \frac{2 n-2 p+3 / 2}{2 n-2 p+1 / 2}\right)^{-1} .
\end{aligned}
$$

or

$$
\left\|\sigma\left(A_{n}\right)\right\|_{G_{p^{n}}}^{2} \geqq(2 \pi)^{-1} \log \left(1+\frac{1}{2 n-2 p+1 / 2}\right) .
$$

Since the $G_{p}^{n}$ 's are disjoint we have

$$
\left\|\sigma\left(A_{n}\right)\right\|^{2} \geqq \sum_{p=1}^{n}\left\|\sigma\left(A_{n}\right)\right\|_{G_{p^{n}}}^{2} \geqq(2 \pi)^{-1} \sum_{p=1}^{n} \log \left(1+\frac{1}{2 n-2 p+1 / 2}\right) .
$$

Therefore

$$
\left\|\sigma\left(A_{n}\right)\right\|^{2} \geqq(2 \pi)^{-1} \sum_{p=0}^{n-1} \log \left(1+\frac{1}{2 p+1 / 2}\right) .
$$

The right hand side of this last inequality becomes infinite as $n \rightarrow \infty$, which completes the proof of the second part.

This last example has further interest in that Pfluger [1] has shown that given $\omega, \sigma \in \Gamma_{h}$, there is a subsequence $n_{j}$ depending on $\omega$ and $\sigma$ such that $\left(\omega,\left(T_{n_{j}} \sigma\right)^{*}\right) \rightarrow\left(\omega, \sigma^{*}\right)$ as $n \rightarrow \infty$.

This last example can be modified to give a canonical homology basis where the vector sum $\mathfrak{A}+\mathfrak{B}$ is not closed in $\Gamma_{h}$. We obtain a new canonical homology basis $A_{1}^{\prime}, B_{1}^{\prime}, A_{2}^{\prime}, B_{2}^{\prime}, \cdots$ from the old as follows: $A_{2 n}^{\prime}=A_{2 n}$, $B_{2 n}^{\prime}=B_{2 n}, A_{2 n-1}^{\prime}=B_{2 n-1}$, and $B_{2 n-1}^{\prime}=-A_{2 n-1}$. If $\mathfrak{P}^{\prime}$ is the span of the $\sigma\left(A^{\prime}\right)^{\prime} \mathrm{s}$ and $\mathfrak{B}^{\prime}$ is the span of the $\sigma\left(B^{\prime}\right)$ 's then $\mathfrak{A}^{\prime}+\mathfrak{B}^{\prime}$ is not closed, for 


$$
\left\|\sigma\left(A_{2 n}^{\prime}\right)-\sigma\left(B_{2 n-1}^{\prime}\right)\right\|
$$

is bounded but $\left\|\sigma\left(A_{2 n}^{\prime}\right)\right\| \rightarrow \infty$. Therefore the projection maps on $\mathfrak{A}^{\prime}$ and $\mathfrak{B}^{\prime}$ cannot be continuous. Since Pfluger's result shows that the finite bilinear relation holds with respect to this new canonical homology basis, we see that the converse of Theorem 5 of $\S I I$ is not true.

\section{BibLIOGRAPHY}

AHLFORS, L.

1. Normalintegrale auf offenen Riemannschen Flächen, Ann. Acad. Sci. Fenn. Ser. A. I. no. 35 (1947).

2. The method of orthogonal decomposition for differentials on open Riemann surfaces, Ann. Acad. Sci. Fenn. Ser. A. I. no. 249/7 (1958).

3. The theory of square integrable differentials on open Riemann surfaces, Report to the Mathematics Division, Air Force Office of Scientific Research, ARDC, Washington 25, KARLIN, S.

D. C., AFOSR Report Number TN 57 790, January 1958.

1. Bases in Banach spaces, Duke Math. J. vol. 15 no. 4 (1958) pp. 971-985. KusUNOKI, Y.

1. On Riemann's period relations on open Riemann surfaces, Mem. Coll. Sci. Univ. Kyoto Ser. A. Math. vol. 30 no. 1 (1956).

Nevanlinna, R.

1. Quadratisch integrierbare Differentiale auf einer Riemannschen Mannigfaltigkeit, Ann. Acad. Sci. Fenn. Ser A. I. no. 1 (1941).

Pfluger, A.

1. Über die Riemannische Periodenrelation auf transzendenten hyperelliptischen Flächen, Comm. Math. Helv. vol. 30 (1956) pp. 98-106.

2. Theorie der Riemannschen Flächen, Berlin, Springer Verlag, 1957. Virtanen, K. I.

1. Über Abelsche Integrale auf nullberandeten Riemannschen Flächen von unendlichem Geschlecht, Ann. Acad. Sci. Fenn. Ser. A. I. no. 56 (1949).

HARVARD UNIVERSITY,

Cambridge, Massachusetts 